\title{
Uso de Misoprostol Retal para Indução do Parto em Gestantes com Amniorrexe Prematura: Ensaio Clinico fase II
}

\author{
Use of Rectal Misoprostol for Induction of Labor in Patients with Premature \\ Rupture of Membranes: a Phase II Clinical Trial
}

Francisco Carlos Nogueira Arcanjo ${ }^{1}$, Carlos Augusto Alencar Júnior ${ }^{2}$, Francisco Edson de Lucena Feitosa ${ }^{2}$, Melania Maria Ramos de Amorim $^{3}$

\begin{abstract}
RESUMO
Objetivo: avaliar se o misoprostol por via retal constitui método efetivo para indução do parto em gestantes com amniorrexe prematura a termo.

Métodos: realizou-se estudo piloto, incluindo 32 gestantes com amniorrexe prematura entre 36 e 41 semanas, feto vivo e único, em apresentação cefálica, escore de Bishop $\leq 6$ e sem contrações de trabalho de parto. Todas receberam misoprostol retal (comprimidos de 50 $\mu$ g) a cada 4 horas até deflagração do trabalho de parto. Pacientes com tempo de bolsa rota $\geq 18$ horas receberam antibiótico (penicilina cristalina) para profilaxia de infecção estreptocócica. Analisaram-se desfechos diversos como intervalo entre indução e início do trabalho de parto, entre indução e parto, incidência de taquissistolia, tipo de parto, incidência de corioamnionite e resultados neonatais. A análise estatística foi realizada no programa de domínio público Epi-Info 2002, calculando-se médias com os respectivos desvios-padrão, além de distribuições de freqüência. Realizou-se análise de sobrevivência para determinação do percentual de partos em função do tempo transcorrido (em horas) desde a administração do primeiro comprimido.

Resultados: os intervalos (média \pm desvio padrão) entre indução e início das contrações $e$

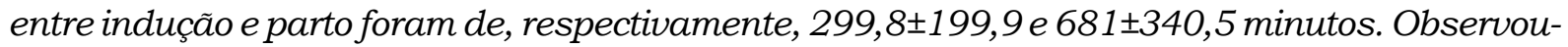
se freqüência de 9,4\% de taquissistolia. Cerca de $72 \%$ das pacientes evoluíram para parto vaginal. Diagnosticou-se corioamnionite em 12,5\% dos casos. As medianas dos escores de Apgar foram de 8 e 9 no primeiro e quinto minuto, respectivamente. Não houve nenhum caso de Apgar $<7$ no quinto minuto. Sepse foi constatada em 12,5\% dos recém-nascidos.

Conclusões: a indução do parto com misoprostol retal foi efetiva em pacientes com amniorrexe prematura, constatando-se $72 \%$ de partos vaginais e baixa freqüencia de corioamnionite. Estes achados precisam ser confirmados em grandes ensaios clínicos controlados.
\end{abstract}

PALAVRAS-CHAVE: Indução do parto. Prostaglandinas. Trabalho de parto.

${ }^{1}$ Maternidade Sant'Ana da Santa Casa de Misericórdia de Sobral - Ceará

${ }^{2}$ Maternidade Escola Assis Chateaubriand - Universidade Federal do Ceará

${ }^{3}$ Instituto de Saúde Elpídio de Almeida - Universidade Federal de Campina Grande e Instituto Materno-Infantil de Pernambuco (IMIP) - Recife Correspondência:

Melania Maria Ramos de Amorim

Rua Neuza Borborema de Sousa, 300 - Bairro Santo Antônio

58103-313 - Campina Grande - PB

e-mail: melamorim@uol.com.br

\section{Introdução}

A amniorrexe prematura ocorre em aproximadamente $10 \%$ das gestações a termo, associando-se a aumento do risco de infecção materna e perinatal, à medida que aumenta o tempo transcorrido entre a ruptura das membranas e o parto ${ }^{1}$. Desta forma, a conduta atualmente preconizada para gestações a termo é a indução do parto se o 
trabalho de parto não se inicia espontaneamente logo depois da amniorrexe ${ }^{2-4}$.

Entretanto, como as condições cervicais são freqüentemente desfavoráveis nestas pacientes, a indução com ocitocina pode ser falha, sobrelevando assim as taxas de operação cesariana. Métodos que propiciem o preparo cervical são, portanto, mais indicados, como o misoprostol e as prostaglandinas, associando-se com maior probabilidade de indução bem sucedida e redução significativa da duração do trabalho de parto ${ }^{5}$.

O misoprostol é um éster sintético da prostaglandina $E_{1}$, cuja eficácia para preparo cervical e indução do parto já foi demonstrada em numerosos ensaios clínicos controlados ${ }^{6}$. Duas revisões sistemáticas estão disponíveis na Biblioteca Cochrane, avaliando o uso do misoprostol por via oral $^{7}$ e vaginal ${ }^{8}$. Esta última incluiu 62 ensaios clínicos randomizados, nos quais o uso de misoprostol foi comparado com placebo e com métodos diversos como prostaglandina $\mathrm{E}_{2}$ vaginal ou intracervical e ocitocina. A conclusão foi que o uso do misoprostol associa-se com maior freqüência de parto vaginal dentro de 24 horas, menor intervalo até o início das contrações e até o parto, acarretando, porém, acréscimo da síndrome de hiperestimulação uterina. Revisões sistemáticas de estudos comparando ambas as vias ainda não estão disponiveis. Existem, porém, metanálises comparando as diferentes doses. Na revisão de Sanchez-Ramos et al. ${ }^{9}$, comparou-se a dose vaginal de $25 \mu \mathrm{g}$ com $50 \mu \mathrm{g}$ em cinco ensaios clínicos randomizados, encontrando-se maior risco de hiperestimulação com a dose de $50 \mu \mathrm{g}$, que, entretanto, associou-se a menor intervalo até o parto e maior proporção de partos vaginais dentro de 24 horas. Os resultados perinatais foram semelhantes para os dois esquemas. Doses menores $(12,5 \mu \mathrm{g})$ têm sido testadas em estudos mais recentes ${ }^{10}$, porém as evidências são insuficientes para se recomendar sua utilização.

O uso do misoprostol em pacientes com amniorrexe prematura também foi estudado em diversos ensaios clínicos, avaliando tanto a via oral como a via vaginal, em posologias variando de $25 \mu \mathrm{g}$ até $100 \mu \mathrm{g}^{11-17}$. Em um estudo não cego, comparando o uso do misoprostol intravaginal com a conduta expectante em casos de amniorrexe prematura a termo, Özden et al. ${ }^{11}$ descreveram, no grupo das pacientes que receberam misoprostol, intervalo significativamente menor até o parto, menor necessidade de uso da ocitocina e desfechos neonatais semelhantes. No estudo de Shetty et al. ${ }^{12}$, observou-se também taxa mais elevada de partos vaginais dentro de 24 horas entre as pacientes que receberam misoprostol oral $(72 \%)$ em relação às pacientes mantidas em conduta expectante $(26,9 \%)$, além do que o grau de satisfação com a conduta adotada foi significativamente maior no grupo do misoprostol. Dois estudos controlados compararam misoprostol oral com placebo, encontrando resultados semelhantes ${ }^{13,14}$.

Ensaios clínicos randomizados comparando o uso do misoprostol oral ${ }^{5}$ ou vaginal ${ }^{15-17}$ com ocitocina ou gel de prostaglandina (dinoprostona) em pacientes com bolsa rota foram também publicados, demonstrando menor intervalo até o parto nos casos submetidos à indução com misoprostol e, em geral, maior proporção de partos vaginais em 12 ou 24 horas. A maioria destes estudos evidenciou maior freqüência de taquissistolia em pacientes que receberam misoprostol, embora esta não tenha se associado a complicações maternas e perinatais. Por outro lado, no estudo de Wing e Paul $^{16}$, não houve diferença significativa entre misoprostol vaginal $(25 \mu \mathrm{g})$ e ocitocina quanto ao intervalo até o parto, proporção de partos vaginais, freqüência de taquissistolia e de corioamnionite.

Uma preocupação com o uso do misoprostol por via vaginal nos casos de ruptura prematura das membranas é o risco teórico de infecção ascendente durante a aplicação dos comprimidos, podendo aumentar as taxas de corioamnionite e sepse neonatal. Este risco não tem sido adequadamente avaliado nos estudos em pacientes com amniorrexe. $\mathrm{Na}$ maioria destes estudos, não se determinou a freqüência de infecção materna e neonatal, além do que o poder da amostra era insuficiente para evidenciar eventuais diferenças ${ }^{11-}$ 17. Os desfechos primários estudados incluíram, em geral, o intervalo até o parto e o percentual de partos vaginais em 24 horas. Wing e Paul ${ }^{16}$ encontraram freqüência semelhante de corioamnionite quando compararam misoprostol vaginal e ocitocina ( 28,6 e $26 \%$, respectivamente). Além disso, nenhum estudo comparou misoprostol oral e vaginal em pacientes com amniorrexe. Entretanto, revisão sistemática comparando prostaglandina vaginal com ocitocina para indução do parto na presença de ruptura prematura das membranas a termo evidenciou maior risco de corioamnionite nas mulheres que receberam prostaglandina ${ }^{18}$. No estudo do TERMPROM de Hannah et al. ${ }^{1}$ também observou-se taxa de corioamnionite mais elevada associada ao uso da prostaglandina vaginal em relação à ocitocina.

Nos últimos anos, a utilização do misoprostol por via retal tem sido proposta para prevenção ou tratamento de hipotonia uterina e hemorragia pósparto $^{19,20}$. Estudo de farmacocinética publicado recentemente evidenciou um melhor perfil farmacocinético do misoprostol retal em relação ao oral, com menor pico sérico, porém maior duração da concentração máxima, o que resultou em 
maior área sob a curva até 240 minutos $^{21}$. Entretanto, até onde chega nosso conhecimento, nenhum estudo avaliou a possibilidade de uso do misoprostol retal para indução do parto.

Em revisão da literatura incluindo os bancos de dados Medline, Embase e Lilacs, além de pesquisa na Cochrane, utilizando os termos "misoprostol" and "rectal" and "labor", não se identificaram estudos, observacionais ou ensaios clínicos, avaliando a administração retal do misoprostol para indução do trabalho de parto. No Registro de Ensaios Controlados da Colaboração Cochrane também não se encontrou referência a esta indicação que, ao nosso ver, teria especial interesse nos casos de amniorrexe prematura, para minimizar os riscos de infecção decorrentes da manipulação vaginal.

Desta forma, considerando que inexistem dados de literatura pertinentes ao uso do misoprostol via retal para indução do parto, realizamos estudo piloto com o objetivo de determinar a efetividade desta via em pacientes com amniorrexe prematura, para definir a viabilidade de um ensaio clínico randomizado (fase III).

\section{Métodos}

Realizou-se estudo piloto, que consistiu de ensaio clínico aberto, fase II, envolvendo 32 gestantes com amniorrexe prematura em quatro hospitais do Nordeste do Brasil: Maternidade da Santa Casa de Misericórdia de Sobral, MaternidadeEscola Assis Chateaubriand (Universidade Federal do Ceará), Instituto Materno-Infantil de Pernambuco (IMIP) e Instituto de Saúde Elpídio de Almeida (Universidade Federal de Campina Grande). Este estudo respeitou as normas da Declaração de Helsinque e da resolução n ${ }^{196 / 96}$ do Conselho Nacional de Saúde para pesquisa em seres humanos. O projeto que deu origem à pesquisa foi aprovado pelos Comitês de Ética em Pesquisa das respectivas instituições. Todas as pacientes foram devidamente informadas sobre os objetivos da pesquisa e concordaram em participar do estudo, assinando o termo de consentimento livre e esclarecido. A pesquisa foi realizada no período de janeiro a julho de 2003.

Em se tratando de estudo piloto, e desconhecendo-se estudos prévios de utilização do misoprostol por via retal para indução do trabalho de parto, decidiu-se arbitrariamente pela inclusão de 30 mulheres, sendo que ao final foram incluídas mais duas. Somente a partir dos dados encontrados nesta série será possível o cálculo do tamanho da amostra para um ensaio clínico randomizado, comparando o misoprostol retal com outras vias (oral, vaginal) em pacientes com amniorrexe prematura.

Foram incluídos gestantes com diagnóstico firmado de amniorrexe prematura entre 37 e 41 semanas, escore de Bishop desfavorável $(\leq 6)$, feto vivo e único com boa vitalidade, em apresentação cefálica de vértice, na ausência de contrações uterinas características do trabalho de parto. Foram excluídas os casos com cicatriz uterina prévia (cesárea ou outras cirurgias), malformações fetais, diagnóstico de corioamnionite ou líquido amniótico meconial na admissão e todas as pacientes com intercorrências associadas, como anemia, sindromes hipertensivas, herpes genital, diabetes e outras morbidades. Além destas, também foram excluídas as gestantes com discordância maior que duas semanas entre a idade gestacional estimada pela amenorréia e a ultra-sonografia.

Amniorrexe prematura foi diagnosticada pela constatação, no exame médico da admissão, do escoamento de líquido amniótico através do orificio cervical externo, durante o exame especular. A idade gestacional foi determinada a partir da data da última menstruação, quando conhecida e confiável, e/ou por ultra-sonografia, considerando-se para fins de cálculo o exame mais precoce (até 20 semanas). Para diagnóstico de vitalidade fetal, realizou-se ausculta fetal com sonar Doppler na admissão, incluindo-se apenas os conceptos com freqüência cardiaca fetal entre 120 e 160 bpm, sem desacelerações detectáveis durante pelo menos cinco minutos de ausculta. Todas as pacientes com tempo de bolsa rota igual ou superior a 18 horas receberam penicilina cristalina na dose inicial de 5.000.000 UI, seguindo-se 2.500.000 UI por via intravenosa a cada 4 horas, para profilaxia da infecção por Streptococcus $\beta$-haemolyticus do grupo B.

A administração do misoprostol foi realizada pelo médico plantonista, introduzindo-se dois comprimidos de $25 \mu \mathrm{g}$ por via retal. Estes comprimidos são comercialmente disponíveis sob o nome fantasia de Prostokos ${ }^{\circledR}$ (Hebron), sendo originalmente preparados para administração vaginal. A seguir, as pacientes foram acompanhadas, monitorizando-se a presença de contrações uterinas a cada hora, até o parto. Esta monitorização foi realizada clinicamente em todos os casos, sendo que a cardiotocografia não foi empregada de rotina. A dose do misoprostol foi repetida a cada quatro horas, somente nos casos em que não se obteve padrão contrátil satisfatório. Considerou-se padrão contrátil satisfatório a presença de pelo menos três contrações de 40 ou mais segundos de duração, em 10 minutos de observação. Obtendo-se este padrão de atividade uterina, cessava a administração do misoprostol. 
Analisaram-se as seguintes variáveis: características demográficas das pacientes (idade, idade gestacional, paridade, escore de Bishop inicial, tempo de bolsa rota), intervalo transcorrido entre a administração do primeiro comprimido e o início de padrão contrátil satisfatório, intervalo entre o início deste padrão contrátil e o parto, intervalo entre o primeiro comprimido e o parto, dose total de misoprostol necessária $(\mathrm{mg})$; freqüência de taquissistolia, alterações da freqüência cardiaca fetal, síndrome de hiperestimulação, eliminação de mecônio intraparto, freqüência de parto vaginal, fórceps e diagnóstico de corioamnionite. Além destas, pesquisaram-se variáveis neonatais: peso (em gramas), escores de Apgar no primeiro e no quinto minuto, necessidade de reanimação neonatal na sala de parto, admissão em unidade de terapia intensiva (UTI) neonatal, necessidade de oxigenoterapia, sepse neonatal e óbito.

Uma vez deflagrado o trabalho de parto, procedeu-se à monitorização da dinâmica uterina e da freqüência cardiaca fetal. A ausculta fetal foi realizada de forma intermitente em todos os casos, a cada 15 minutos, antes, durante e depois das contrações, quando presentes. A dinâmica era verificada clinicamente, a cada 30 minutos. Considerou-se taquissistolia a presença de seis ou mais contrações em 10 minutos, por dois períodos consecutivos de 10 minutos. As alterações da freqüência cardiaca fetal consideradas foram taquicardia (acima de $160 \mathrm{bpm}$ ), bradicardia (abaixo de $110 \mathrm{bpm}$ ), desacelerações tardias (DIPS tipo II) ou umbilicais desfavoráveis. Considerou-se como sindrome de hiperestimulação a associação de taquissistolia com essas alterações da freqüência cardíaca fetal. Anotou-se, ainda, a presença ou não de mecônio no líquido amniótico, durante o trabalho de parto, incluindo o período expulsivo, registrando-se suas características (mecônio fluido ou espesso).

O tratamento da taquissistolia foi realizado com nifedipina na dosagem de $20 \mathrm{mg}$ por via sublingual, repetindo-se a cada 30 minutos, se necessário. Além disto, preconizava-se a adoção de decúbito lateral esquerdo e oxigenoterapia materna $\left(\mathrm{O}_{2}\right.$ sob cateter, 5 litros / minuto).

Corioamnionite foi diagnosticada clinicamente com base na presença de febre materna $\left(\geq 38^{\circ} \mathrm{C}\right)$ não atribuível a outras causas, podendo associar-se a taquicardia materna $(>120 \mathrm{bpm})$, taquicardia fetal $(>160 \mathrm{bpm})$, sensibilidade uterina e odor fétido do liquido amniótico.

$\mathrm{O}$ atendimento ao recém-nascido na sala de parto seguiu as normas preconizadas em cada instituição. Registrou-se a necessidade de manobras de reanimação na sala de parto, bem como a admissão na UTI neonatal e oxigenoterapia. Consi- derou-se como oxigenoterapia a utilização de qualquer uma das técnicas de suporte ventilatório (CPAP nasal, ou ventilação mecânica assistida). A sepse neonatal foi definida a partir da evidência clínica de processo infeccioso, com sintomas e sinais inespecíficos acompanhados de duas ou mais alterações laboratoriais e antibioticoterapia concorrente por sete ou mais dias.

A análise estatística foi realizada utilizando-se o programa de domínio público Epi-Info, versão 2002 para Windows. Para análise das características demográficas, dos parâmetros de evolução do trabalho de parto e das variáveis neonatais, obteve-se distribuição de freqüência das variáveis categóricas e medidas de tendência central (média e mediana) e de dispersão (desvio padrão, percentis) das variáveis numéricas. Utilizaramse média e desvios padrão para as variáveis contínuas com distribuição normal (comprovada com o teste de Kolmogorov-Smirnov) e medianas para as variáveis ordinais e sem distribuição normal. Realizou-se análise de sobrevivência (Kaplan-Meier) para determinação do percentual de partos em função do tempo transcorrido (em horas) desde a administração do primeiro comprimido.

\section{Resultados}

A média de idade das pacientes foi em torno de 25 anos, com mediana da paridade de zero, observando-se percentual de $56,2 \%$ de nuliparas. A idade gestacional variou entre 37 e 41 semanas, com média em torno de 39 semanas. Os escores de Bishop iniciais variaram entre zero e seis, com mediana de quatro. Em relação ao tempo de bolsa rota, este variou entre zero (ruptura com menos de uma hora de duração) e 96 horas, com mediana de 7,5 horas (Tabela 1).

A indução foi bem sucedida, verificando-se o desencadeamento de contrações uterinas, em todos os casos. A média do intervalo entre a primeira dose de misoprostol e o início das contrações uterinas foi de 300 minutos (variando entre 60 e 900 minutos), entre o início das contrações e o parto foi de 381 minutos (variando entre 55 e 900 minutos) e entre a primeira dose e o parto, de 681 minutos (variando entre 235 e 1800 minutos). A dose total de misoprostol utilizada variou entre $50 \mu \mathrm{g}$ (uma aplicação) e $250 \mu \mathrm{g}$ (cinco aplicações), com mediana de $100 \mu \mathrm{g}$ (duas aplicações) (Tabela 2).

Dez pacientes $(31,2 \%)$ apresentaram desencadeamento do trabalho de parto com somente uma dose, catorze pacientes $(43,8 \%)$ com duas doses, sete pacientes $(21,9 \%)$ com três doses e apenas uma paciente $(3,1 \%)$ requereu cinco doses. 
Tabela 1 - Características demográficas das pacientes com amniorrexe prematura submetidas à indução do parto com $50 \mu \mathrm{g}$ de misoprostol por via retal.

\section{Característica}

Idade em anos (média \pm DP)

$24,9 \pm 6,6$

(variação: 18-41)

Paridade (mediana)

0

Nulíparas (n, \%)

(variação: 0-10)

$18(56,2 \%)$

Idade gestacional em semanas (variação)

Idade gestacional em semanas (média \pm DP)

$37-41$ semanas

$38,9 \pm 1,6$

$0-6$

4

$0-99$

Tempo de bolsa rota (variação em horas)

7,5 horas

Tempo de bolsa rota (mediana)

Total $=32$ pacientes

Tabela 2 - Evolução do trabalho de parto das pacientes com amniorrexe prematura após administração de $50 \mu \mathrm{g}$ de misoprostol por via retal.

\begin{tabular}{|c|c|}
\hline $\begin{array}{l}\text { Intervalo (minutos) entre a primeira dose e o } \\
\text { início das contrações (média } \pm \text { DP) }\end{array}$ & $\begin{array}{c}299,8 \pm 199,9 \\
\text { (variação: 60-900) }\end{array}$ \\
\hline $\begin{array}{l}\text { Intervalo (minutos) entre o início das } \\
\text { contrações e o parto (média } \pm \text { DP) }\end{array}$ & $\begin{array}{c}381,3 \pm 221,5 \\
\text { (variação: } 55-900 \text { ) }\end{array}$ \\
\hline $\begin{array}{l}\text { Intervalo (minutos) entre a primeira dose e o } \\
\text { parto (média } \pm D P \text { ) }\end{array}$ & $\begin{array}{c}681,0 \pm 340,5 \\
\text { (variação: } 235-1800 \text { ) }\end{array}$ \\
\hline $\begin{array}{l}\text { Dose total do misoprostol }(\mu \mathrm{g}) \text { administrada } \\
\text { (mediana) }\end{array}$ & $\begin{array}{c}100 \mathrm{mg} \\
\text { (variação: } 50-250)\end{array}$ \\
\hline Taquissistolia (n, \%) & $3(9,4 \%)$ \\
\hline Alterações da FCF (n, \%) & $1(3,1 \%)$ \\
\hline Síndrome de hiperestimulação (n, \%) & - \\
\hline Mecônio (n, \%) & $4(12,5 \%)$ \\
\hline Parto vaginal $(n, \%)$ & $23(71,9 \%)$ \\
\hline Fórceps $(\mathrm{n}, \%)$ & - \\
\hline Corioamnionite (n, \%) & $4(12,5 \%)$ \\
\hline Total = 32 pacientes & \\
\hline
\end{tabular}

A freqüência de taquissistolia foi de 9,4\%, porém não se observou nenhum caso de sindrome de hiperestimulação. Houve um caso em que se detectaram padrões anômalos de freqüência cardiaca fetal $(3,1 \%)$, porém sem associação com taquissistolia. Constatou-se a presença de mecônio no líquido amniótico em quatro casos $(12,5 \%)$, nenhum destes associado a alterações da freqüência cardiaca fetal. Cerca de $72 \%$ dos casos ( $n=23$ ) evoluíram para parto vaginal. Quatro pacientes $(12,5 \%)$ tiveram diagnóstico de corioamnionite (Tabela 2).
Realizaram-se nove cesarianas $(28 \%)$, indicadas por distocia (quatro casos), desproporção céfalo-pélvica (dois casos), mecônio espesso no início do trabalho de parto (dois casos) e sofrimento fetal agudo (um caso).

Na Figura 1 apresenta-se a porcentagem acumulada de casos que tiveram o parto assistido (vaginal ou cesárea) em função do intervalo (em horas) transcorrido desde a administração da primeira dose de misoprostol. O tempo necessário para que ocorresse o parto em aproximadamente $90 \%$ das gestantes foi de 18 horas.

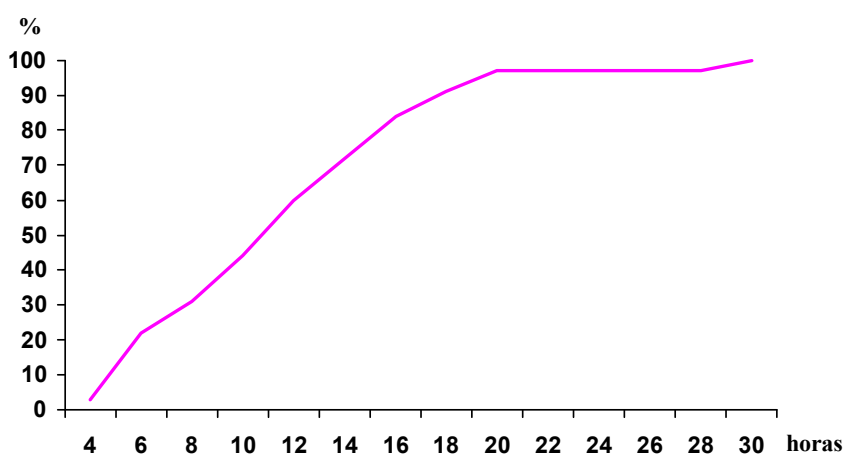

Figura 1 - Porcentagem acumulada de partos em pacientes com amniorrexe prematura após administração do misoprostol retal, em função do tempo (horas).

Em relação aos desfechos neonatais, observou-se média de peso ao nascer em torno de 3.170 $\mathrm{g}$, variando entre 2.365 e $4.080 \mathrm{~g}$. As medianas dos escores de Apgar para o primeiro e o quinto minuto foram de, respectivamente, oito e nove, encontrando-se percentual de 15,6\% de escores de Apgar abaixo de sete no primeiro minuto. Não houve casos de escores de Apgar menores que sete no quinto minuto. Um recém-nascido requereu manobras de reanimação, incluindo entubação endotraqueal $(3,1 \%)$, e dois foram admitidos em UTI neonatal $(6,2 \%)$. Oxigenoterapia suplementar foi administrada a quatro recém-nascidos $(12,5 \%)$. Houve quatro casos de sepse neonatal (12,5\%). Não ocorreram óbitos neonatais (Tabela 3 ).

\section{Discussão}

Os resultados do presente estudo sugerem que o misoprostol por via retal pode ser usado para indução do parto em gestantes com amniorrexe prematura, com taxa de sucesso da indução de $100 \%$, associando-se com freqüência de parto vaginal de $72 \%$ e tempo médio entre a dose inicial e o parto em torno de 680 minutos. Na maioria dos casos (66\%), verificou-se o desencadeamento do trabalho de parto com no máximo duas doses de 
misoprostol. Encontrou-se baixa freqüência de taquissistolia $(9,4 \%)$ e os resultados perinatais foram favoráveis, não se observando escores de Apgar menores que sete no quinto minuto. A freqüência de corioamnionite e infecção neonatal foi de $12,5 \%$.

Tabela 3 - Desfechos neonatais em pacientes com amniorrexe prematura submetidas à indução do parto com $50 \mu \mathrm{g}$ de misoprostol por via retal.

\section{Desfecho neonatal}

Peso ao nascer em gramas (média \pm DP)

$3172,4 \pm 411$

(variação: $2365-4080$ )

Apgar de primeiro minuto (mediana)

8

(variação: $3-9$ )

9

Apgar de quinto minuto (mediana)

(variação: 7-10)

Apgar $<7$ no primeiro minuto (n, \%)

$5(15,6 \%)$

Apgar $<7$ no quinto minuto (n, \%)

Necessidade de reanimação neonatal $(n, \%)$

Admissão em UTI neonatal ( $\mathrm{n}, \%)$

$2(6,2 \%)$

Necessidade de oxigenoterapia ( $n, \%)$

$4(12,5 \%)$

Sepse neonatal $(n, \%)$

$4(12,5 \%)$

Óbito neonatal $(\mathrm{n}, \%)$

Total $=32$ recém-nascidos

Esses resultados não são surpreendentes, uma vez que a efetividade do misoprostol para indução do parto por outras vias (oral, vaginal, sublingual) tem sido demonstrada em diversos ensaios clínicos e revisões sistemáticas. Evidências nivel I destacam a superioridade da droga em relação à maioria dos métodos convencionais de indução do parto, sobretudo quando as condições cervicais são desfavoráveis ${ }^{7,8}$. A utilização em casos de amniorrexe prematura também já foi descrita em diversos ensaios clínicos controlados, sugerindo efeitos favoráveis tanto em comparação com placebo como com ocitocina ${ }^{11-17,22}$.

Por outro lado, a utilização do misoprostol via retal tem sido amplamente estudada com o objetivo de prevenir ou tratar hipotonia uterina e hemorragia pós-parto, desde 1998, quando foi publicado o primeiro relato com esta proposta ${ }^{23}$. Seguiram-se diversos ensaios clínicos randomizados ${ }^{24-26}$ e, em 2003, uma revisão sistemática publicada na Cochrane avaliou a efetividade do misoprostol para o tratamento da hemorragia pós-parto primária $^{20}$. Na verdade, um único ensaio clínico, sulafricano, foi incluído na metanálise, envolvendo 64 mulheres com perda sanguínea pós-parto acima de $500 \mathrm{~mL}^{25}$. Nestas pacientes, o misoprostol apresentou melhores resultados que os ocitócicos (infusão intravenosa ou instilação miometrial) para reduzir a perda sanguínea $(\mathrm{OR}=0,18$; IC 95\% $=0,04-0,67)$. A conclusão dos revisores foi que o misoprostol retal na dose de $800 \mu \mathrm{g}$ pode representar opção de primeira linha para o tratamento de hemorragia pós-parto primária.

A farmacocinética do misoprostol via retal também já foi avaliada em estudo que considerou seu uso no terceiro estágio do trabalho de parto. Nesse estudo, descreveram-se para o misoprostol retal, em relação ao oral, menor pico sérico, intervalo mais prolongado até atingir-se a concentração sérica máxima e maior área sob a curva, além de menor freqüência de efeitos colaterais ${ }^{21}$. Essas características são semelhantes às da via vaginal, porém não existem estudos comparando a farmacocinética das duas vias. Mesmo assim, a via retal surge como alternativa viável quando a via vaginal não pode ser utilizada, como nos casos de sangramento genital profuso.

Até onde chega nosso conhecimento, este estudo é pioneiro na utilização do misoprostol via retal para indução do parto. Revisando tanto o banco de dados Medline como o Lilacs/Scielo, não encontramos qualquer referência na literatura pertinente sobre o uso de misoprostol com essa finalidade. Entretanto, nossos resultados se apóiam em base farmacológica bem definida e são comparáveis com os achados de outros estudos usando misoprostol por outras vias em pacientes com amniorrexe prematura.

A freqüência de parto vaginal $(72 \%)$ foi semelhante à descrita por Shetty et al. ${ }^{12}$, porém diferiu da encontrada por diversos outros autores $^{5,15,16}$, que descreveram taxas mais elevadas de parto vaginal (em torno de $90 \%$ ) em pacientes com ruptura prematura das membranas. Julgamos, na verdade, que eventuais diferenças entre o escore cervical na admissão entre os estudos podem justificar este achado, uma vez que incluimos apenas pacientes com cérvice desfavorável. Na revisão sistemática da Cochrane ${ }^{8}$, quando se consideraram apenas os estudos comparando misoprostol vaginal e ocitocina em todas as pacientes com bolsa rota e cérvice variável ou não definida, encontrou-se taxa de parto vaginal de $89 \%$, porém quando foram analisados os estudos em pacientes com escore cervical desfavorável esta freqüência foi em torno de $80 \%$. Outra explicação razoável parte do pressuposto de que taxas diferentes de cesariana são descritas em diversos países, refletindo discrepâncias da conduta obstétrica adotada nas diversas instituições.

De qualquer forma, como encontramos taxa de sucesso na indução em todos os casos, essa diferença na taxa de parto vaginal em relação a outros estudos publicados não parece ser 
indicativa de menor efetividade do misoprostol retal. As principais indicações de cesariana foram distocia e desproporção céfalo-pélvica, e todas as cesarianas foram indicadas em pacientes sem distúrbios da contratilidade uterina, em trabalho de parto com dilatação avançada. Por outro lado, para determinar se realmente existem diferenças na freqüência de cesariana com o uso do misoprostol retal, seria necessário um grupo-controle com a utilização de misoprostol por outras vias já consagradas (oral, vaginal).

Em relação ao tempo transcorrido entre o início da indução (administração da primeira dose) e o parto verificado no presente estudo (681 minutos), este se assemelhou ao encontrado por outros autores ${ }^{8,22}$, destacando-se a rápida evolução destas pacientes, uma vez desencadeado o trabalho de parto.

Diversos estudos avaliaram o intervalo entre indução e parto em pacientes com amniorrexe, considerando diferentes vias e esquemas de misoprostol. Ngai et al. ${ }^{27}$ registraram tempo médio de 7,5 horas utilizando misoprostol oral na dose de $200 \mathrm{mg}$, porém esta dose é considerada atualmente muito elevada, associando-se a aumento do risco de hiperestimulação ${ }^{16,28}$. Em outro estudo usando misoprostol oral na dose de $50 \mu \mathrm{g}$ a cada quatro horas em pacientes com amniorrexe prematura, Butt et al. ${ }^{22}$ descreveram média de intervalo entre indução e parto de 720 minutos nas pacientes que receberam misoprostol, porém este tempo foi mais longo que aquele observado entre as pacientes recebendo ocitocina (501 minutos), principalmente devido a intervalo mais longo entre a indução e o início das contrações. Já em nosso estudo, encontramos intervalo bem menor entre indução e início das contrações, em torno de 300 minutos.

Acreditamos que a principal vantagem da via retal em pacientes com ruptura prematura das membranas, além da segurança e efetividade, é que permite evitar toques vaginais repetidos, o que teoricamente reduziria o risco de corioamnionite. $\mathrm{Na}$ verdade, encontramos freqüência muito baixa tanto de infecção materna como neonatal $(12,5 \%)$, sobretudo quando se consideram os índices encontrados por outros autores nos casos de amniorrexe submetidos à indução do parto com misoprostol vaginal, em torno de $29 \%{ }^{16}$. Todavia, esta redução precisa ser comprovada em ensaios clínicos controlados, comparando a via retal com a via vaginal.

Encontramos freqüência de taquissistolia bem mais baixa $(9,4 \%)$ que a descrita na literatura. Sanchez-Ramos et al. ${ }^{15}$ descreveram freqüência em torno de $29 \%$, ao passo que Ngai et al. ${ }^{27}$ encontraram freqüência em torno de $31 \%$ em pacientes com amniorrexe prematura. Taxas entre
$20 \%$ e $30 \%$ são descritas na maioria dos estudos em pacientes submetidas a indução do trabalho de parto com misoprostol, independente da presença ou não de membranas rotas ${ }^{7,8}$. Entretanto, How et al. ${ }^{29}$ relataram freqüência mais baixa de hiperestimulação quando se utilizou misoprostol oral na dose de $25 \mu \mathrm{g}(4 \%)$, em relação à mesma dose administrada por via vaginal (15\%). No estudo de Fisher et $a .^{30}$, a taxa de taquissistolia também foi maior entre pacientes recebendo misoprostol oral na dose de $50 \mu \mathrm{g}(9,7 \%)$, quando comparadas àquelas que receberam misoprostol vaginal $(26,5 \%)$. No entanto, a possibilidade de menor freqüência de taquissistolia com o misoprostol retal deve ser testada em grandes estudos randomizados analisando maior número de pacientes com as diferentes vias.

Analisando os desfechos perinatais, estes foram semelhantes aos descritos na literatura em pacientes com amniorrexe prematura ${ }^{15,16,27}$; o percentual de escores de Apgar inferior a sete foi de $15,6 \%$ no primeiro minuto, porém nenhum caso foi observado no quinto minuto. A revisão sistemática publicada por Hofmeyr e Gulmezoglu ${ }^{8}$ descreveu freqüência global de 1,4\% de baixos escores de Apgar no quinto minuto nos casos de bolsa rota submetidos à indução com misoprostol vaginal. A freqüência de eliminação de mecônio intraparto $(12,5 \%)$ também é compativel com os resultados obtidos por outros autores utilizando misoprostol oral e vaginal em pacientes com e sem bolsa rota ${ }^{7,8}$.

O presente estudo aponta para a possibilidade de uso do misoprostol por via retal para indução do parto. Evidentemente, em se tratando de estudo piloto, não controlado, são inequivocas as limitações metodológicas, e os resultados aqui encontrados valem basicamente como ponto de partida para futuros ensaios clínicos randomizados, confrontando o misoprostol retal com outros métodos de indução (como a ocitocina) e outras vias e esquemas de misoprostol.

Acreditamos que o misoprostol retal tenha potencial indicação em pacientes com ruptura prematura das membranas, com o possivel beneficio de redução das taxas de infecção materna e neonatal, porque reduz os riscos da manipulação vaginal. Em relação ao misoprostol oral, que também poderia ser usado com essa finalidade, os benefícios advêm do perfil farmacocinético mais favorável, tanto pelo menor pico sérico como pela maior área sob a curva ${ }^{21}$. Entretanto, tornam-se necessárias evidências mais confiáveis, nível I, que só podem ser obtidas em ensaios clínicos controlados, comparando o misoprostol retal com outras vias (oral, vaginal) em pacientes com ruptura prematura das membranas. 
Neste sentido, estamos elaborando o projeto de um grande ensaio clínico multicêntrico, com três braços (misoprostol oral, vaginal e retal) em pacientes com amniorrexe prematura, devendo utilizar os resultados preliminares aqui obtidos para cálculo do tamanho da amostra. Vale a pena ressaltar que o poder deverá ser suficiente para demonstrar diferenças pertinentes tanto ao intervalo indução-parto como à via de parto e, principalmente, à freqüência das complicações infecciosas (corioamnionite, sepse neonatal), que não têm sido adequadamente avaliadas (desfechos secundários) em estudos semelhantes.

Por outro lado, devem-se analisar as expectativas e o grau de satisfação das mulheres envolvidas nessas pesquisas, uma vez que a aceitação da via retal pode representar um problema na prática clínica, quando se considera o uso para indução do parto.

\section{ABSTRACT}

Purpose: to investigate whether rectally administered misoprostol is an effective method for induction of labor in patients with premature ruptured membranes at term.

Methods: a pilot trial was conducted, enrolling 32 women with alive, singleton, cephalic fetus and ruptured membranes between 36 and 41 weeks of pregnancy, with Bishop score $\leq$ 6 and without evidence of labor. They received rectal misoprostol (tablets of $50 \mu \mathrm{g}$ ) every $4 \mathrm{~h}$ until active labor was diagnosed. Patients with ruptured membranes for $\geq 18 \mathrm{~h}$ received antibiotics (crystalline penicillin) for prophylaxis of streptococcal infeccion. Outcomes included time from induction to labor and induction to delivery, incidence of tachysystole, mode of delivery, incidence of chorioamnionitis and neonatal outcome. Statistical analysis was performed using the public domain software Epi-Info 2002. Means and standard deviations were calculated, as well as frequency distributions. Survival analysis was performed to determine percent of deliveries according to time (hours) since the administration of the first tablet.

Results: the mean $( \pm S D)$ induction-to-labor and inductionto-delivery intervals were $299.8 \pm 199.9$ and $681 \pm 340.5 \mathrm{~min}$, respectively. The frequency of tachysystole was $9.4 \%$. About $72 \%$ of patients achieved vaginal delivery. Chorioamnionitis was diagnosed in $12.5 \%$ of the patients. Median Apgar scores at 1 st and 5th min were 8 and 9 , respectively. There was no case of Apgar $<7$ at the 5th min. Neonatal sepsis occurred in $12.5 \%$ of the neonates.

Conclusions: induction of labor with rectal misoprostol in the setting of premature rupture of membranes was effective, with $72 \%$ of vaginal deliveries and a low rate of chorioamnionitis. These findings must be confirmed by large randomized controlled trials.

KEYWORDS: Labor induction. Prostaglandins. Labor.
Conflito de interesses: os comprimidos de misoprostol utilizados nesta pesquisa foram fornecidos pelo laboratório Hebron. Não houve financiamento de outra espécie. Nenhum dos pesquisadores recebeu bolsas pelo trabalho.

\section{Referências}

1. Hannah ME, Ohlsson A, Farine D, et al. Induction of labor compared with expectant management for prelabor rupture of the membranes at term. TERMPROM Study Group. N Engl J Med 1996; 334:1005-10.

2. Natale R, Milne JK, Campbell MK, Potts PG, Webster K, Halinda E. Management of premature rupture of membranes at term: randomized trial. Am J Obstet Gynecol 1994; 171:936-9.

3. Mozurkewich EL, Wolf FM. Premature rupture of membranes at term: a meta-analysis of three management schemes. Obstet Gynecol 1997; 89:1035-43.

4. ACOG practice bulletin. Premature rupture of membranes. Clinical management guidelines for obstetrician-gynecologists. Number 1, June 1998. American College of Obstetricians and Gynecologists. Int J Gynaecol Obstet 1998; 63:75-84.

5. Ngai SW, Chan YM, Lam SW, Lao TT. Labour characteristics and uterine activity: misoprostol compared with oxytocin in women at term with prelabour rupture of the membranes. BJOG 2000; 107:222-7.

6. Wing DA. Labor induction with misoprostol. Am J Obstet Gynecol 1999; 181:339-45.

7. Alfirevic Z. Oral misoprostol for induction of labour. Cochrane Database Syst Rev 2003; (2):CD00395.

8. Hofmeyr GJ, Gulmezoglu AM. Vaginal misoprostol for cervical ripening and induction of labour. Cochrane Database Syst Rev 2003; (2):CD00032.

9. Sanchez-Ramos L, Kaunitz AM, Delke I. Labor induction with 25 microg versus 50 microg intravaginal misoprostol: a systematic review. Obstet Gynecol 2002; 99:145-51.

10.Tedesco RP, Cecatti JG, Maia Filho, NL. Efetividade de duas diferentes doses de misoprostol por via vaginal para preparo cervical e indução do parto. Rev Bras Ginecol Obstet 2002; 24:641-6.

11.Özden S, Delikara MN, Avci A, Ficicioglu C. .Intravaginal misoprostol vs. expectant management in premature rupture of membranes with low Bishop scores at term. Int $\mathrm{J}$ Gynaecol Obstet 2002; 77:109-15. 
12.Shetty A, Stewart K, Stewart G, Rice P, Danielian $\mathrm{P}$, Templeton A. Active management of term prelabour rupture of membranes with oral misoprostol. BJOG 2002; 109:1354-8.

13.Hoffmann RA, Anthony J, Fawcus S. Oral misoprostol vs. placebo in the management of prelabor rupture of membranes at term. Int J Gynaecol Obstet 2001; 72:215-21.

14.Lo JY, Alexander JM, McIntire DD, Leveno KJ. Ruptured membranes at term: randomized, doubleblind trial of oral misoprostol for labor induction. Obstet Gynecol 2003; 101:685-9.

15.Sanchez-Ramos L, Delke I. Induction of labor with misoprostol for premature rupture of membranes beyond thirty-six weeks' gestation. Am J Obstet Gynecol 1999; 180:253-4.

16.Wing DA, Paul RH. Induction of labor with misoprostol for premature rupture of membranes beyond thirty-six weeks' gestation. Am J Obstet Gynecol 1998; 179:94-9.

17.Frohn WE, Simmons S, Carlan SJ. Prostaglandin E2 gel versus misoprostol for cervical ripening in patients with premature rupture of membranes after 34 weeks. Obstet Gynecol 2002; 99:206-10.

18.Tan BP, Hannah ME. Prostaglandins versus oxytocin for prelabour rupture of membranes at or near term. Cochrane Database Syst Rev 2000; (2):CD000158.

19.Villar J, Gulmezoglu AM, Hofmeyr GJ, Forna F. Systematic review of randomized controlled trials of misoprostol to prevent postpartum hemorrhage. Obstet Gynecol 2002; 100:1301-12.

20. Mousa HA, Alfirevic Z. Treatment for primary postpartum haemorrhage. Cochrane Database Syst Rev 2003; (2):CD02131.

21.Khan RU, El-Refaey H. Pharmacokinetics and adverse-effect profile of rectally administered misoprostol in the third stage of labor. Obstet Gynecol 2003; 101:968-74.
22.Butt KD, Bennett KA, Crane JM, Hutchens D, Young DC. Randomized comparison of oral misoprostol and oxytocin for labor induction in term prelabor membrane rupture. Obstet Gynecol 1999; 94:9949.

23.O'Brien P, El-Refaey H, Gordon A, Geary M, Rodeck $\mathrm{CH}$. Rectally administered misoprostol for the treatment of postpartum hemorrhage unresponsive to oxytocin and ergometrine: a descriptive study. Obstet Gynecol 1998; 92:212-4.

24.Bamigboye AA, Hofmeyr GJ, Merrell DA. Rectal misoprostol in the prevention of postpartum hemorrhage: a placebo-controlled trial. Am J Obstet Gynecol 1998; 179:1043-6.

25.Lokugamage AU, Sullivan KR, Niculescu I, et al. A randomized study comparing rectally administered misoprostol versus Syntometrine combined with an oxytocin infusion for the cessation of primary post partum hemorrhage. Acta Obstet Gynecol Scand 2001; 80:835-9.

26. Bugalho A, Daniel A, Faundes A, Cunha M. Misoprostol for prevention of postpartum hemorrhage. Int J Gynaecol Obstet 2001; 73:1-6.

27.Ngai SW, To WK, Lao T, Ho PC. Cervical priming with oral misoprostol in pre-labor rupture of membranes at term. Obstet Gynecol 1996; 87:923-6.

28.Adair CD, Weeks JW, Barrilleaux S, Edwards M, Burlison K, Lewis DF. Oral or vaginal misoprostol administration for induction of labor: a randomized, double-blind trial. Obstet Gynecol 1998; 92:810-3.

29. How HY, Leaseburge L, Khoury JC, Siddiqi TA, Spinnato JA, Sibai BM. A comparison of various routes and dosages of misoprostol for cervical ripening and the induction of labor. Am J Obstet Gynecol 2001; 185:911-5.

30.Fisher SA, Mackenzie VP, Davies GA. Oral versus vaginal misoprostol for induction of labor: a doubleblind randomized controlled trial. Am J Obstet Gynecol 2001; 185:906-10.

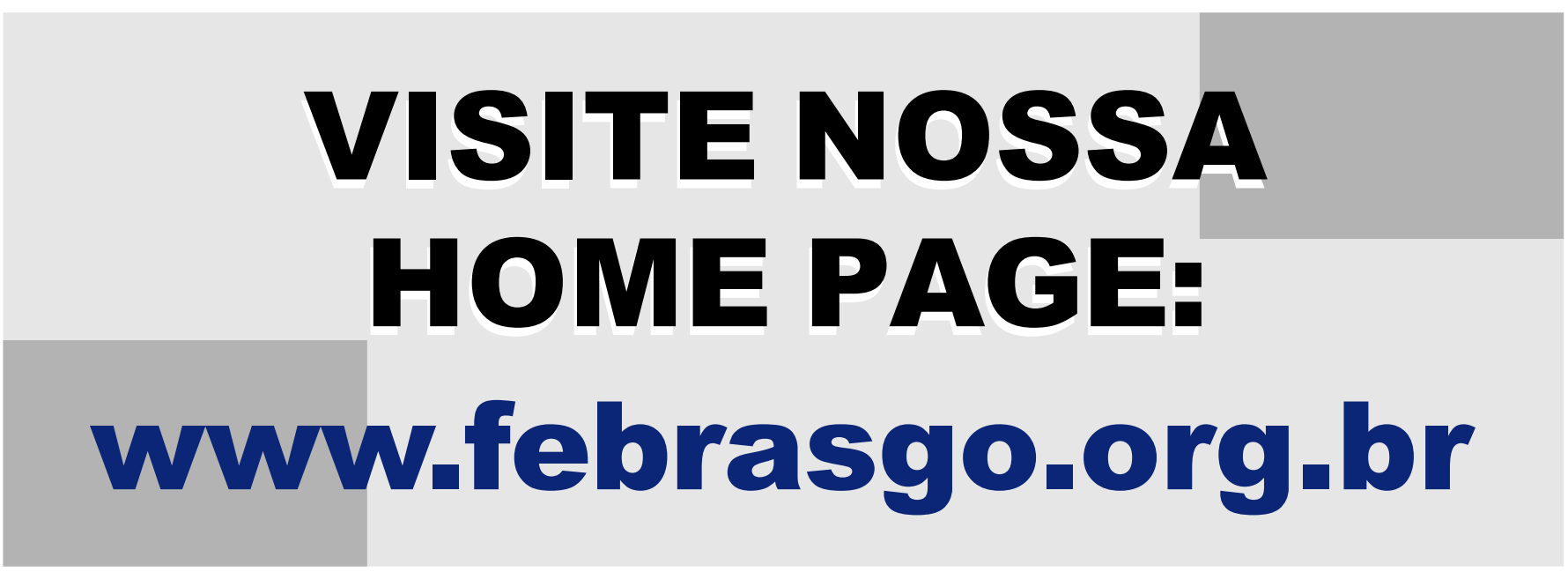

